\title{
From autoimmune thyroid disease and CREST syndrome to incidental discovery of incipient gastric carcinoma: a case report
}

\author{
Lisandra Damian ${ }^{*}, 1,2$ Maria Christina Ungureanu², Cristina Mihaela Ghiciuc ${ }^{1}$, \\ Francesca Romana Patacchioli ${ }^{3}$, Lucia Corina Dima-Cozma ${ }^{4}$, Sebastian Cozma ${ }^{5}$, \\ Cătălina Elena Lupușoru ${ }^{1}$
}

1Department of Pharmacology, "Grigore T. Popa" University of Medicine and Pharmacy, lași,

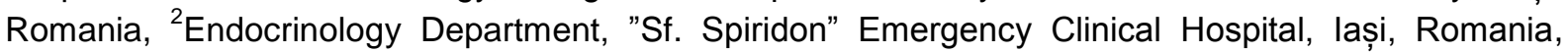
${ }^{3}$ Department of Physiology and Pharmacology "V. Erspamer", Sapienza University of Rome, Italy, ${ }^{4}$ Department of Internal Medicine, "Grigore T. Popa" University of Medicine and Pharmacy, lași, Romania, ${ }^{5}$ Department of Otorhinolaryngology, "Grigore T. Popa", University of Medicine and Pharmacy, lași, Romania.

\begin{abstract}
Autoimmune thyroid diseases (AITDs), including Graves' Disease and Hashimoto's Thyroiditis (chronic autoimmune thyroiditis), are the most common organ-specific autoimmune disorders, occurring mostly in middleaged women. AITDs are often associated with other autoimmune diseases such as vitiligo or systemic sclerosis (scleroderma), but there have been only few reports of AITDs concurring with CREST syndrome (Calcinosis, Raynaud's phenomenon, Esophageal dysmotility, Sclerodactyly and Telangiectasias), a limited form of scleroderma. Furthermore, these disorders have been associated with lymphoid malignancies, but only rarely with other cancers such as lung or breast.

We report the case of a 59 year-old female patient who presented at the Endocrinology clinic for vertigo, dysphoria and fatigue. Clinical examination and serologic determinations revealed the diagnosis of an overlap syndrome between euthyroid autoimmune thyroiditis and CREST syndrome. Further investigations led to the detection of an incipient gastric adenocarcinoma which was promptly treated with total gastrectomy.
\end{abstract}

Keywords: autoimmune thyroid disease, CREST syndrome, scleroderma, gastric carcinoma

\section{Introduction}

Autoimmune thyroid disease (AITD), including Graves' disease and Hashimoto's Thyroiditis (chronic autoimmune thyroiditis), is a well-documented clinical entity and the most common organ-specific autoimmune disorder, occurring mostly in middle-aged women [1-3].

Received: April 2015; Accepted after review: June 2015; Published: June 2015.

${ }^{*}$ Corresponding author: Lisandra Damian MD, Department of Pharmacology, "Grigore T. Popa", University of Medicine and Pharmacy, 16 Universitatii Str., lași, 700115, Romania;

E-mail: lisandra.damian@gmail.com
The main characteristic of the disease is the lymphocytic infiltration of the thyroid gland and the presence of circulating anti-thyroid antibodies (anti-thyroperoxidase, anti-thyroid stimulating hormone, anti-thyroglobulin) [4].

AITDs often concur with other autoimmune diseases, giving rise to the concept of overlap syndromes, which are defined as the coexistence of two or more autoimmune disorders in one individual $[5,6]$.

Previous studies have reported the association between AITDs and systemic scleroderma, an autoimmune connective tissue disorder characterized by systemic inflammation, microvascular abnormalities, and fibrosis of the skin and internal organs [7-10]. 
However, there have been only few reports of AITDs associated with CREST syndrome (Calcinosis, Raynaud's phenomenon, Esophageal dysmotility, Sclerodactyly and Telangiectasias), a limited form of scleroderma [11].

Furthermore, these disorders have been associated with lymphoid malignancies [12, 13], but only rarely with other cancers such as lung, breast, duodenum [14-16].

We herein report a case of incipient gastric adenocarcinoma uncovered during the evaluation of overlap syndrome between autoimmune thyroiditis and CREST syndrome.

\section{Case Report}

We report the case of a 59 year-old female patient who came to the Endocrinology Clinic for vertigo, dysphoria, and fatigue.

The patient's history revealed that she suffered from Raynaud's phenomenon for the past ten years, for which she did not seek medical attention. She was hypertensive for approximately seven years, currently under treatment with angiotensin II receptor blockers (ARBs) and calcium channel blockers (CCBs).

The patient had a family history of cardiovascular diseases and metabolic syndrome, but no autoimmune diseases or malignancies were reported.

On admission to our clinic the patient was overweight (with a BMI of $28.1 \mathrm{~kg} / \mathrm{m}^{2}$ ), with normal pulmonary and cardiovascular parameters, a resting heart rate of $70 \mathrm{bpm}$ and a blood pressure of $150 / 100 \mathrm{mmHg}$.

Clinical examination revealed: erythema of the face, thin lips, erased expression lines, mild hardening of the skin, telangiectasias (Figure 1) of the lips, face and palms, sclerodactyly (Figure 2), non-pitting edema of the lower limbs, subcutaneous calcinosis (Figure 3 ) in the extensor surface of the joints (elbow and knee), bilaterally and mildly impaired strength prehension.

Laboratory findings showed normal blood cell count, mild elevations of the sedimentation rate $(16 \mathrm{~mm} / \mathrm{h})$, high total cholesterol (268 $\mathrm{mg} / \mathrm{dl})$, LDL cholesterol $(180 \mathrm{mg} / \mathrm{dl})$ and triglycerides $(232 \mathrm{mg} / \mathrm{dl})$. Renal and hepatic parameters were within normal limits, while glucose levels were slightly elevated (107 $\mathrm{mg} / \mathrm{dl})$.

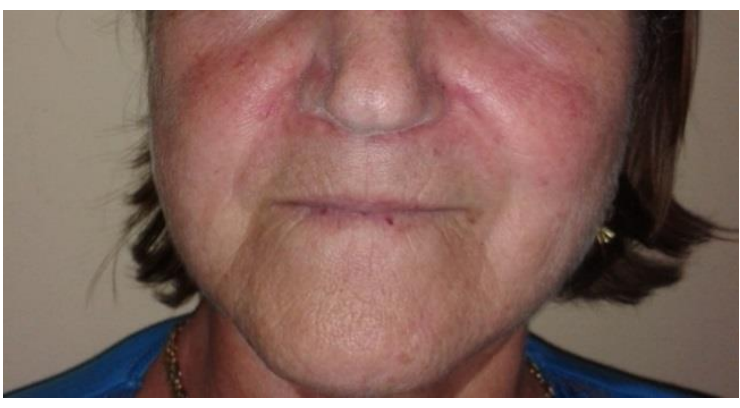

Fig. 1. Telangiectasias of the face and lips

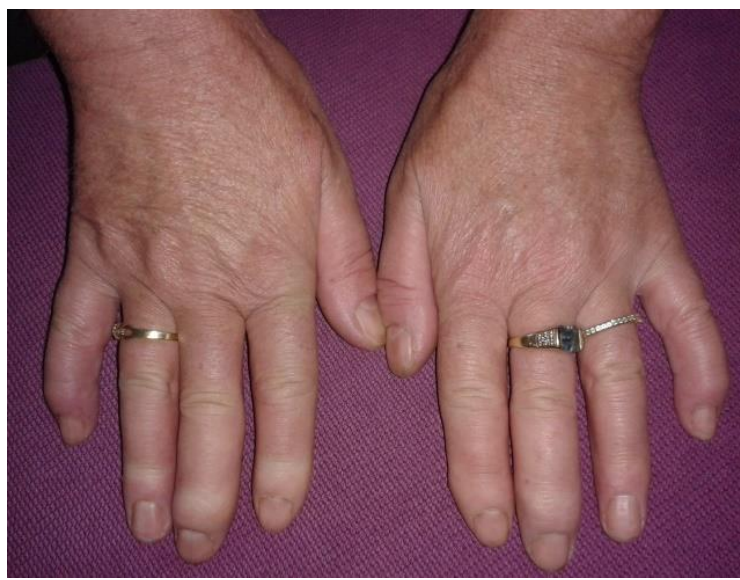

Fig. 2. Sclerodactyly
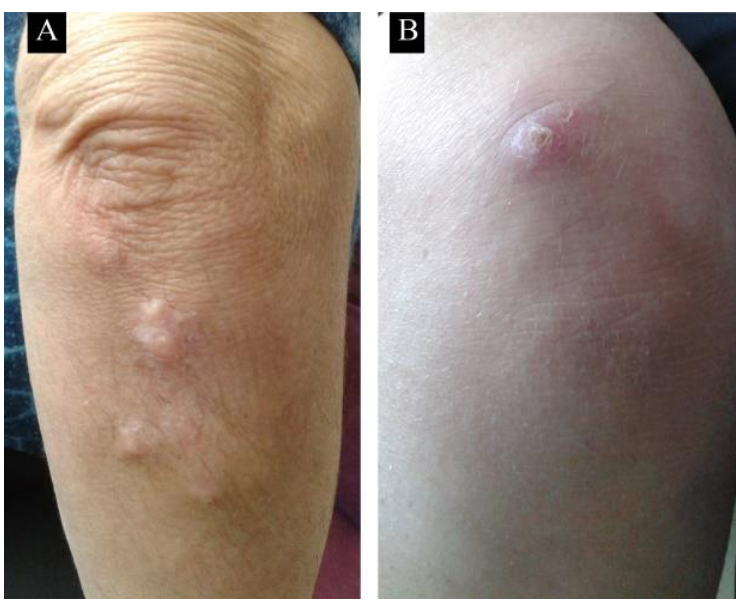

Fig. 3. Calcinosis cutis of the elbow (A) and knee (B)

Immunological assays revealed autoimmune thyroiditis (high levels of antithyroperoxidase antibodies) with euthyroid status (normal thyroid stimulating hormone and free thyroxin levels) and the presence of antinuclear antibodies (ANA), with high titers of anti-centromere antibodies $(675 \mathrm{Ul} / \mathrm{ml})$ and anti-chromatin antibodies (243 Ul/ml), but negative Scl-70 antibodies. 
Neck ultrasound revealed a small thyroid parenchyma (total volume of $6.6 \mathrm{ml}$ ), highly heterogeneous, with multiple discrete hypoechoic micronodules and normal vascularity, which is strongly suggestive for chronic thyroiditis.

With the purpose of investigating the patient's main symptom (severe vertigo) we recommended a neurological exam which did not reveal any pathological elements and the brain MRI did not find any cerebral lesions.

Electrocardiogram at rest was normal and the 2D transthoracic Doppler echocardiography showed concentric left ventricular hypertrophy, normal and symmetrical contractions with a normal ejection fraction of $68 \%$. Doppler ultrasound of the carotid arteries revealed atherosclerosis with calcified plaques at the origin of the left internal carotid artery, with a $20 \%$ stenosis.

Cervical spine $x$-ray showed an inverted physiological curvature at the C3-C4 level, with arthrosis and a narrowing of the spinal canal at the C4-C7 level.

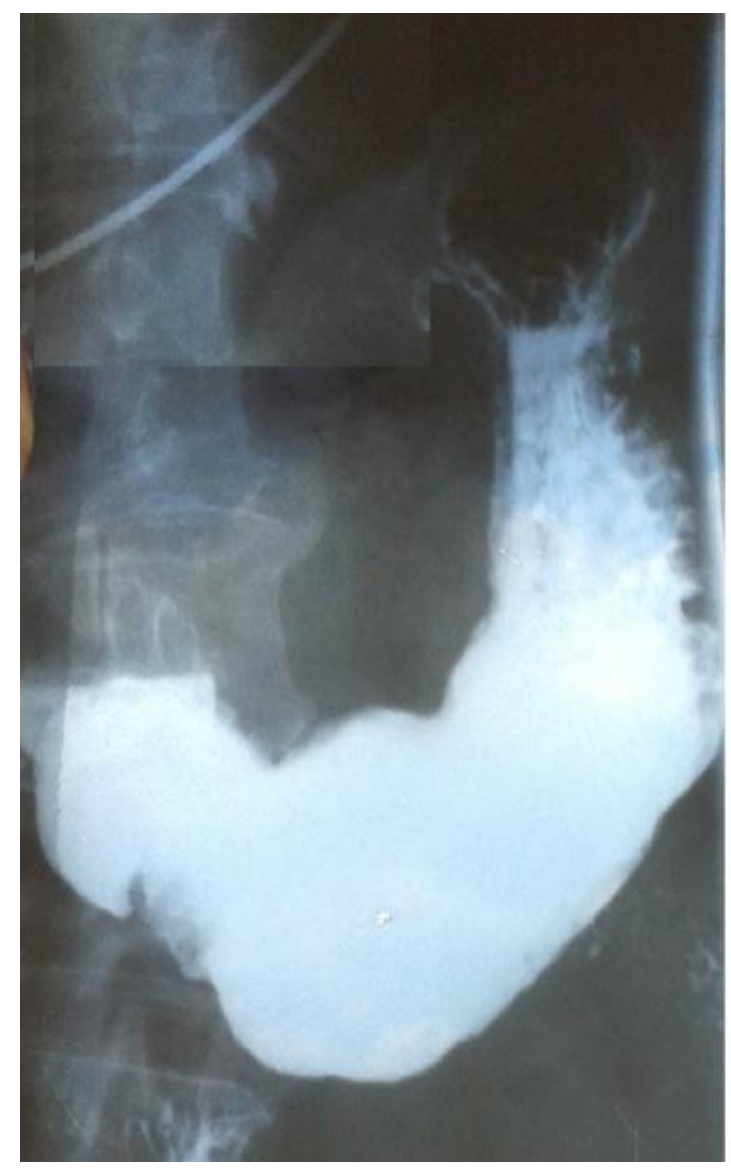

The otorhinolaryngology examination found a normal audiogram, normal caloricvestibular test, negative head-shake, therefore could not establish an organic cause for the symptoms.

Based on the clinical picture (calcinosis, Raynaud's phenomenon, sclerodactyly and telangiectasias) and immunological findings (high levels of anti-centromere and antichromatin antibodies), a diagnosis of CREST syndrome was made and cyclosporine treatment was recommended.

To further evaluate any esophageal involvement of the scleroderma, an esogastric radioscopy was performed, which visualized two lacunar images in the pyloric antrum and hypertrophic gastric folds (Figure 4).

Upper gastrointestinal endoscopy revealed on the greater gastric curvature two ulcerated polypoid tumors with an apparent common base of $1.5-2 \mathrm{~mm}$ and biopsy from the tumors found intramucosal gastric adenocarcinoma.

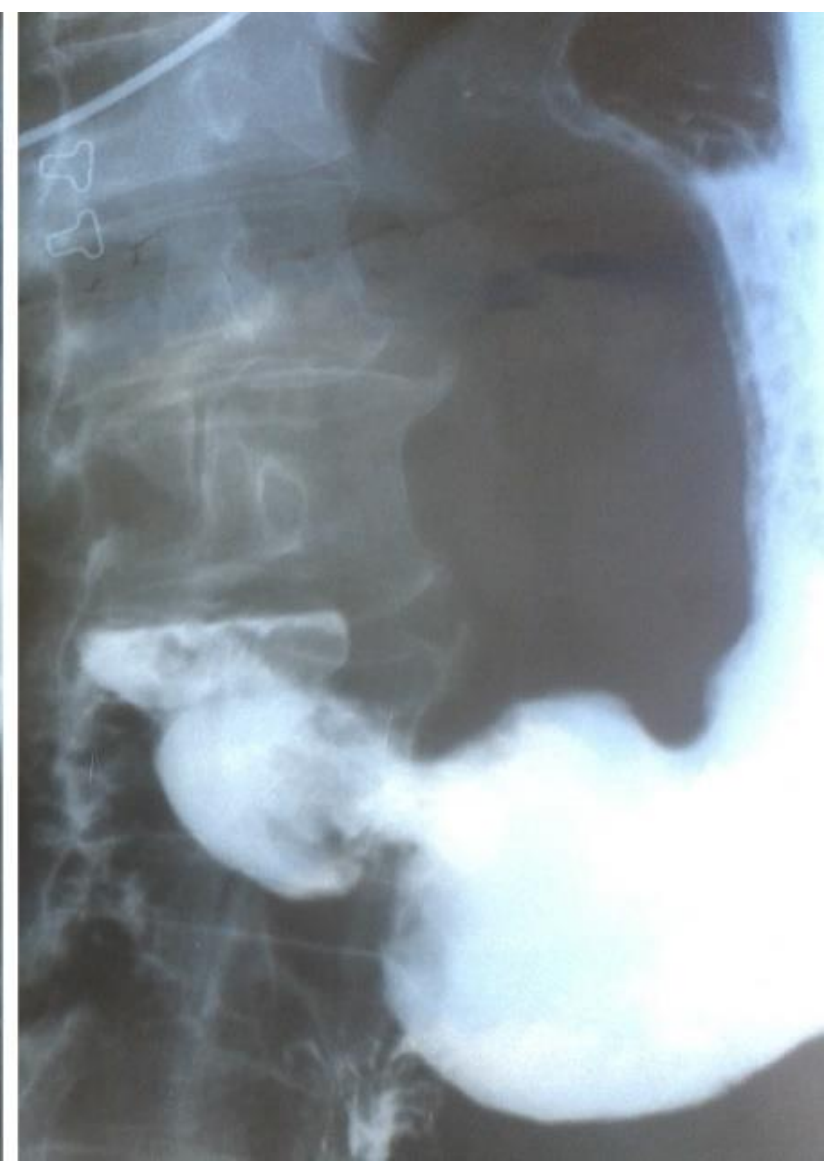

Fig. 4. Gastroscopy - Lacunar images in the pyloric antrum 
The patient was then directed to the Surgery Department of the Regional Institute of Oncology lași, where a total gastrectomy was performed, with a "roux-en-y" anastomosis (termino-lateral eso-jejunoanastomosis and termino-lateral jejuno-jejunoanastomosis).

Pathology report revealed a welldifferentiated tubular adenocarcinoma with superficial submucosal infiltration - $\mathrm{pT}_{1 \mathrm{~b}} \mathrm{~N}_{0} \mathrm{M}_{1}$.

The patient had a favorable postoperative evolution and the prognosis is good due to the very early diagnosis of the gastric malignancy.

However, taking into consideration this recent diagnosis, the immunosuppressive treatment with cyclosporine for CREST syndrome was deferred.

\section{Discussions}

AITDs are the most common autoimmune disorders in humans and have a higher prevalence in the female population, women: men ratio of $4: 1[3,17,18]$. AITDs have been reported to be commonly associated with other autoimmune diseases such as vitiligo or systemic sclerosis (scleroderma), but only rarely with CREST syndrome [19, 20].

Studies have shown that the association of autoimmune disorders is more than coincidental, identifying a common genetic background and an autoimmune underlying mechanism [6, 21].

Several publications informed of a possible increased risk of malignancies in scleroderma, particularly lung, breast, lymphoid and hematological neoplasms [14].

A few older papers suggested a possible link between gastric carcinoma and systemic sclerosis, on an atrophic gastritis and

\section{References}

1. Swain M, Swain T, Mohanty BK. Autoimmune thyroid disorders - an update. Indian J Clin Biochem 2005; 20(1):9-17.

2. Fountoulakis $S$, Tsatsoulis $A$. On the pathogenesis of autoimmune thyroid disease: a unifying hypothesis. Clin Endocrinol (Oxf) 2004; 60:397-409.

3. Merrill SJ, Mu Y. Thyroid autoimmunity as a window to autoimmunity: an explanation for sex
Helicobacter pylori (H. pylori) background, which were strongly associated with esophageal hypomotility [22]. However, our patient had hypertrophic gastric folds on gastroscopy and was negative for $\mathrm{H}$. pylori.

Our case presentation confirms the possibility of overlap syndrome between autoimmune thyroiditis and CREST syndrome and highlights the importance of a multidisciplinary approach of the patient with autoimmune disorders.

Moreover, the serologic confirmation of CREST syndrome in a patient without any digestive symptoms could have masked the presence of the gastric malignancy.

The screening for esophageal dysmotility in our patient allowed for the early diagnosis of an otherwise silent disease, frequently discovered in the complications stages.

\section{Conclusions}

In summary, thyroid and connective tissue autoimmune diseases may overlap with each other and together may mask the existence of malignancies.

It is important to be aware that CREST syndrome and autoimmune thyroiditis can coexist and a thorough evaluation of both conditions and gastric cancer screening may be necessary even in patients who do not complain with digestive symptoms.

\section{Acknowledgements}

This paper was published under the frame of European Social Fund, Human Resources Development Operational Program 2007-2013, project no. POSDRU/159/1.5/S/136893. 
rheumatic disease. J Clin Pathol 2001; 54(5):340-347.

6. Sung SJ, Hwang SJ, Kim JM, Lee YA, Oh JS, Suh $\mathrm{JH}$, et al. A case of polymyositis associated with Hashimoto's thyroiditis. Korean $J$ Intern Med 2013; 28(3):380-382.

7. Antonelli A, Ferri $C$, Fallahi $P$, et al. Clinical and subclinical autoimmune thyroid disorders in systemic sclerosis. Eur J Endocrinol 2007; 156(4):431-437.

8. Elhai $\mathrm{M}$, Avouac J, Kahan A, Allanore $\mathrm{Y}$. Systemic sclerosis at the crossroad of polyautoimmunity. Autoimmun Rev 2013; 12(11):1052-1057.

9. Robazzi TCMV, Adan LFF. Autoimmune thyroid disease in patients with rheumatic diseases. Rev Bras Reumatol 2012; 52(3):417-430.

10. Punzi L, Betterle C. Chronic autoimmune thyroiditis and rheumatic manifestations. Joint Bone Spine 2004; 71(4):275-283.

11. Nakamura $T$, Higashi $S$, Tomoda $K$, Tsukano $M$, Sugi K. Primary biliary cirrhosis (PBC)CREST overlap syndrome with coexistence of Sjögren's syndrome and thyroid dysfunction. Clin Rheumatol 2007; 26(4):596-600.

12. Koyama $T$, Fujimoto $K$, Shima $M$. Acquired von Willebrand syndrome associated with Hashimoto's thyroiditis and subcutaneous mucosa-associated lymphoid tissue lymphoma. Intern Med 2013; 52(23):2661-2663.

13. William BM, Harbert $T$, Ganti AK, Bierman PJ. Small lymphocytic lymphoma in a patient with CREST syndrome. Hematol Oncol Stem Cell Ther 2011; 4(3):132-135.

14. Bonifazi M, Tramacere I, Pomponio G, et al. Systemic sclerosis (scleroderma) and cancer risk: systematic review and meta-analysis of observational studies. Rheumatology (Oxford) 2013; 52(1):143-154.

15. Kounalakis N, Pezner R, Staud CL, Kruper L. Partial breast irradiation in a patient with bilateral breast cancers and CREST syndrome. Brachytherapy 2011; 10(6):486-490.

16. Anastasopoulos G, Marinis A, Konstantinidis C, Theodosopoulos T, Fragulidis G, Vassiliou I. Adenocarcinoma of the third portion of the duodenum in a man with CREST syndrome. World J Surg Oncol 2008; 6:106.

17. Vanderpump MPJ. The epidemiology of thyroid disease. Br Med Bull 2011; 99:39-51.

18. Schott M, Scherbaum WA. Autoimmune thyroid disease. Dtsch Arztblt 2006; 103(45):A30233032.

19. Antonelli A, Fallahi P, Ferrari SM, Mancusi C, Giuggioli D, Colaci M, et al. Incidence of thyroid disorders in systemic sclerosis: results from a longitudinal follow-pp. J Clin Endocrinol Metab 2013; 98(7):E1198-1202.

20. Bonilla-Abadía F, Muñoz-Buitrón E, Ochoa CD, Carrascal E, Cañas CA. A rare association of localized scleroderma type morphea, vitiligo, autoimmune hypothyroidism, pneumonitis, autoimmune thrombocytopenic purpura and central nervous system vasculitis. Case report. BMC Res Notes 2012; 5:689.

21. Yu H, Qiu H, Pan J, Wang S, Bao Y, Jia W. Hashimoto's thyroiditis concomitant with sequential autoimmune hepatitis, chorea and polyserositis: a new entity of autoimmune polyendocrine syndrome? Intern Med 2013; 52(2):255-258.

22. Matsuta M, Chiba $C$, Akasaka $T$, Terashima M. A case of systemic sclerosis associated with gastric cancer. J Dermatol 1999; 26(9):611614. 